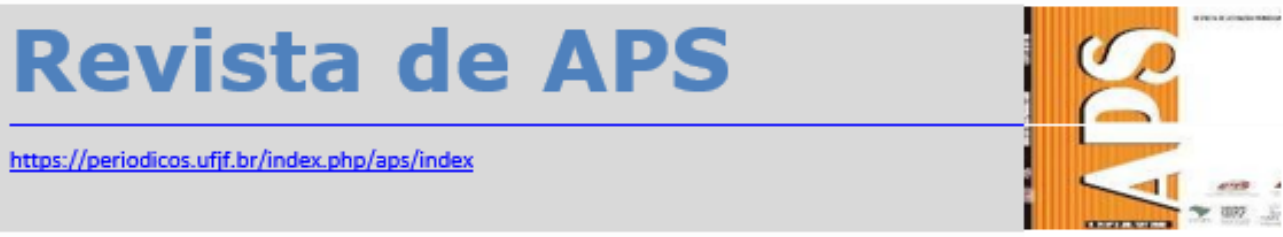

\title{
Planejamento regional no enfrentamento às doenças crônicas
}

\author{
Regional Planning in fighting Chronic Diseases
}

\author{
Bruna Schossler, ${ }^{1}$ Cássia Regina Gotler Medeiros, ${ }^{2}$ Morgana Salvadori, ${ }^{3}$ Olinda Maria \\ de Fátima Lechmann Saldanha ${ }^{4}$
}

\begin{abstract}
RESUMO
O objetivo deste estudo foi analisar o planejamento regional para o enfrentamento das doenças crônicas não transmissíveis (DCNT) na 29a Região de Saúde do Rio Grande do Sul e comparar com as metas nacionais do Plano de Ações Estratégicas para o Enfrentamento das DCNT no Brasil (2011-2022). Pesquisa descritiva, com abordagem quantitativa, por meio de análise documental de pautas e atas de reuniões da Comissão Intergestores Regional (CIR) de 2014 e de cinco planos municipais de saúde do período 2014-2017. Identificaram-se debates relacionados ao enfrentamento das DCNT nos pontos de pauta e atas das reuniões da CIR; contudo, estes são pouco relacionados à promoção da saúde. A análise do diagnóstico situacional dos planos apresentou ausência de informações importantes, como perfil de morbimortalidade e metas relacionadas à gestão, ao monitoramento e à avaliação. Entre os objetivos propostos pelos planos municipais, quatro contemplaram 10 das 12 metas nacionais, com exceção ao consumo de frutas e hortaliças e ao tratamento das lesões precursoras de câncer. Para qualificar o enfrentamento das DCNT, ações relacionadas à promoção da saúde e avaliação precisam integrar o planejamento regional em saúde.
\end{abstract}

PALAVRAS-CHAVE: Doença crônica. Planejamento em saúde. Gestão em saúde. Promoção da saúde.

\section{ABSTRACT}

The objective was to analyze how the regional planning to confront the Non-

\footnotetext{
${ }^{1}$ Centro de Ciências Biológicas e da Saúde da Universidade do Vale do Taquari (Univates).

${ }^{2}$ Centro de Ciências Biológicas e da Saúde da Universidade do Vale do Taquari (Univates). E-mail: cgotlermedeiros@gmail.com.

${ }^{3}$ Centro de Ciências Biológicas e da Saúde da Universidade do Vale do Taquari (Univates).

${ }^{4}$ Centro de Ciências Biológicas e da Saúde da Universidade do Vale do Taquari (Univates).
} 
communicable Chronic Diseases (NCCD) has been done in the 29th Healthcare Region of Rio Grande do Sul state, and compare it with the national goals of the Brazilian Strategic Action Plan to Combat Chronic Non-communicable Diseases (2011-2012). A descriptive research, with a quantitative approach, through documentary analysis of agendas and minutes of meetings of the Regional Interagency Commission (CIR) and five municipal health plans from the period of 2014-2017. Discussions about dealing with NCCD in agendas and minutes of the RIC meetings were identified; however, they are loosely related to health promotion. The analysis of the situational diagnosis of the plans presented a lack of relevant information, such as the profile of morbidity and mortality, and goals regarding management, monitoring and assessment. Among the goals set by the municipal plans, four contemplated 10 out of the 12 national goals, except the consumption of fruits and vegetables, and the treatment of cancer precursor lesions. To qualify fighting NCCD, actions related to health promotion need to integrate regional health planning.

KEYWORDS: Chronic disease. Health planning. Health management. Health promotion.

\section{INTRODUÇÃO}

Nas últimas décadas evidenciou-se mundialmente a transformação do perfil de adoecimento populacional. O aumento da expectativa de vida, a acelerada urbanização e a transição socioeconômica, epidemiológica e nutricional, bem como a ampliação do acesso aos serviços de saúde, elevaram a prevalência de doenças crônicas não transmissíveis (DCNT), criando um novo padrão de morbimortalidade. ${ }^{1}$ Consideradas a principal causa de morte no mundo, em 2008, as DCNT representavam 63\% dos óbitos; no Brasil, 72\%, refletindo principalmente nas camadas vulneráveis da população. ${ }^{2}$

A Organização Mundial da Saúde (OMS) define como principais DCNT: doenças cardiovasculares e respiratórias crônicas, neoplasias e o diabetes mellitus. Seus fatores de risco são classificados em não modificáveis (sexo, idade e herança genética) e comportamentais (tabagismo, alimentação inadequada, inatividade física e consumo elevado de álcool e outras drogas). ${ }^{3}$ Entretanto, é possível diminuir seu impacto mediante intervenções precoces de promoção da saúde, a partir da construção de planos fundados em ações intersetoriais, reconhecendo as peculiaridades de cada espaço e região. ${ }^{4}$

Monitorar os fatores de risco, a morbimortalidade e as respostas dos sistemas de saúde é segundo o Ministério da Saúde (MS), estratégia fundamental para o 
enfrentamento das DCNT. ${ }^{2}$ O planejamento em saúde é imprescindível nos casos em que a complexidade das tarefas, bem como os recursos e as pessoas necessárias para seu desenvolvimento, não podem ser improvisados. ${ }^{5}$ Com a necessidade de organizar as redes de atenção em saúde, uma nova forma de planejar o trabalho é proposta pelo Decreto no 7.508/2011, um processo de planejamento ascendente, integrado e regionalizado. ${ }^{6}$

O Decreto citado contribui para a organização, o planejamento, a assistência à saúde e a articulação interfederativa no Sistema Único de Saúde (SUS). Define as Regiões de Saúde como o espaço geográfico contínuo que integra a organização, o planejamento e a execução de ações e de serviços de saúde; e as Comissões Intergestores Regionais (CIR) como instâncias de negociação consensual entre os entes federativos. ${ }^{6}$ O Plano de Saúde representa uma ferramenta que formaliza o planejamento no âmbito das esferas municipal, estadual e federal, e deve conter prioridades e responsabilidades definidas regionalmente e os compromissos do governo com o setor de saúde no período de quatro anos. $^{7}$

Ao reconhecer as DCNT como problema de grande magnitude na vida da população e parte da agenda de compromissos globais, verifica-se a necessidade de ações planejadas de promoção da saúde. A promoção da saúde implica ampliar a qualidade de vida, prolongá-la e evitar doenças, aumentando assim a autonomia e o bem-estar do indivíduo. ${ }^{8}$

Diante disso, intentou-se investigar o planejamento regional para o enfrentamento das DCNT na 29a Região de Saúde do Rio Grande do Sul e comparar com as metas nacionais do Plano de Ações Estratégicas para o Enfrentamento das DCNT no Brasil (2011-2022). ${ }^{2}$

\section{MÉTODOS}

Estudo realizado por meio de análise documental da Região de Saúde 29, vinculada à 16a Coordenadoria Regional de Saúde (CRS) do Rio Grande do Sul (RS). Tal região possui 209.392 habitantes, ${ }^{9}$ distribuídos em 27 municípios, dos quais 22 têm menos de 10 mil habitantes. Desses municípios, 17 dispõem de profissionais do Programa Mais Médicos, 26 têm Estratégia Saúde da Família (ESF), sendo 20 com 100\% 
de cobertura. ${ }^{10}$

Coletou-se os dados em pautas e atas das reuniões da Comissão Intergestores Regional (CIR) de 2014 (ano anterior ao estudo), e analisou-se cinco Planos Municipais de Saúde (PMS). Selecionou-se os planos de municípios em que houve participação efetiva dos gestores nas reuniões da CIR, conforme observado em lista de presenças. Como critério de inclusão considerou-se o porte populacional, sendo dois municípios com até 5 mil habitantes, um de 5.001 a 10 mil, um de 10.001 até 20 mil, e um com mais de 20 mil habitantes.

O município A tem 20.510 habitantes, e para assistência à saúde da população apresenta uma Unidade Mista, quatro Estratégias Saúde da Família (ESF), cinco Unidades Básicas de Saúde (UBS), um Centro de Atenção Psicossocial (CAPS) e um hospital de pequeno porte. O município B tem 12.320 habitantes, duas ESF e um hospital de pequeno porte. $\mathrm{O}$ município $\mathrm{C}$ conta com uma população de 6.073 habitantes, quatro UBS e uma ESF. O município D tem 2.204 habitantes e uma ESF. O município E conta com 2.030 habitantes e uma ESF.

A análise das informações contidas nas atas e pautas das reuniões da CIR buscou identificar temas relacionados ao enfrentamento das DCNT. Na análise dos PMS o objetivo foi verificar quais, entre as metas nacionais propostas pelo Plano de Ações Estratégicas para o Enfrentamento das DCNT no Brasil no período de 2011-2022, ${ }^{2}$ estavam presentes, e se a análise situacional contemplou dados sociodemográficos, epidemiológicos e de estrutura, assim como relacionados ao planejamento, ao monitoramento e à avaliação.

Organizou-se os resultados em três subseções:

1. Estrutura da análise situacional contemplada nos Planos Municipais de Saúde.

2. Plano de Ações Estratégicas para o Enfrentamento das DCNT no Brasil (20112022)² e Planos Municipais de Saúde.

3. Debates na CIR relacionados às DCNT.

O estudo foi aprovado pelo Comitê de Ética em Pesquisa da Universidade do Vale do Taquari (Univates), sob parecer no 612.126. Destaca-se a concessão da permissão para acesso aos documentos pelos gestores municipais de saúde da 16a CRS/RS, não havendo conflito de interesse dos autores da pesquisa. 


\section{RESULTADOS}

\section{Estrutura da análise situacional contemplada nos planos municipais de saúde}

A análise situacional constitui a base para o desenvolvimento do plano de saúde, pois permite a explicação dos problemas de saúde da população com a intenção de reconhecer as necessidades e elencar as prioridades de atenção. ${ }^{11}$ Para uma análise satisfatória, é necessário conter dados sociodemográficos, epidemiológicos e de estrutura, assim como relacionados ao planejamento, ao monitoramento e à avaliação. Os dados presentes nos PMS estão apresentados no Quadro 1.

Quadro 1 - Estrutura da análise situacional contemplada nos Planos Municipais de Saúde

\begin{tabular}{|l|c|c|c|c|c|}
\hline \multicolumn{1}{|c|}{ Informações } & $\begin{array}{c}\text { Município } \\
\text { A }\end{array}$ & $\begin{array}{c}\text { Município } \\
\text { B }\end{array}$ & $\begin{array}{c}\text { Município } \\
\text { C }\end{array}$ & $\begin{array}{c}\text { Município } \\
\text { D }\end{array}$ & $\begin{array}{c}\text { Município } \\
\text { E }\end{array}$ \\
\hline Dados sociodemográficos & $\mathrm{X}$ & $\mathrm{X}$ & $\mathrm{X}$ & $\mathrm{X}$ & \\
\hline Dados epidemiológicos & $\mathrm{X}$ & & $\mathrm{X}$ & $\mathrm{X}$ & \\
\hline $\begin{array}{l}\text { Planejamento, } \\
\text { monitoramento e avaliação }\end{array}$ & & $\mathrm{X}$ & $\mathrm{X}$ & $\mathrm{X}$ & $\mathrm{X}$ \\
\hline
\end{tabular}

Fonte: Elaborada pelos autores - 2018

Por meio da análise dos PMS observou-se a disposição de dados referentes à população total e à distribuição em área rural e urbana, mencionada pelos municípios A, B e D. Os municípios A e C acrescentaram à análise o crescimento populacional de 1991 a 2010 e a pirâmide etária. Somente o município D apresentou a distribuição da população por raça. Os municípios A, C e D apresentaram valores de PIB e do Índice de Desenvolvimento Humano, este último também apresentado pelo município $B$.

Utilizando o Sistema de Informações de Mortalidade (SIM), os municípios A, C e D apresentaram as principais causas de mortalidade e internações. Ressalta-se que não se encontrou informação sobre os dados epidemiológicos nos PMS dos municípios B e E. Todos descreveram sua estrutura organizacional e os programas desenvolvidos no território. As ações de promoção da saúde envolvem o cuidado em todo o ciclo vital por meio de atendimentos individuais e coletivos, orientados por programas como Rede Cegonha, HiperDia, Programa Saúde na Escola (PSE), Primeira Infância Melhor (PIM), 
Atenção à Saúde Mental e Grupo de Controle ao Tabagismo, descritos no plano de ações estratégicas como fundamentais para o enfrentamento às DCNT no Brasil. ${ }^{2}$

Os municípios C, D e E descreveram como desenvolvem o planejamento, o monitoramento e a avaliação do sistema. O município C citou que utiliza como referência o PMS, as Programações Anuais de Saúde e a Conferência Municipal de Saúde.

O município D realiza o planejamento a partir da análise do perfil epidemiológico da população e da realidade vivenciada pelos profissionais da equipe da ESF. O município $E$ referiu que os profissionais de saúde se reúnem quinzenalmente, fortalecendo o trabalho da equipe. Quanto ao monitoramento e à avaliação, os municípios C e D referiram que a equipe de gestão parte dos indicadores do MS para definir os próximos desafios. O município B declarou que os serviços da área da saúde são avaliados pela população, pelo Conselho Municipal de Saúde e pelos profissionais da área.

Plano de ações estratégicas para o enfrentamento das DCNT no Brasil (2011-2022) e os planos municipais de saúde

O plano de ações estratégicas ${ }^{2}$ está articulado com a Política Nacional de Promoção da Saúde (PNPS), ${ }^{12}$ sendo que 12 das 11 metas propostas referem-se a ações de promoção da saúde, priorizando os principais fatores de risco modificáveis (tabagismo, alimentação inadequada, inatividade física, consumo abusivo de bebidas alcoólicas), conforme consta no Quadro 2.

Quadro 2 - Estratégias de enfrentamento às DCNT propostas nos PMS

\begin{tabular}{|l|c|c|c|c|c|}
\hline Estratégias municipais & $\begin{array}{c}\text { Município } \\
\text { A }\end{array}$ & $\begin{array}{c}\text { Município } \\
\text { B }\end{array}$ & $\begin{array}{c}\text { Município } \\
\text { C }\end{array}$ & $\begin{array}{c}\text { Município } \\
\text { D }\end{array}$ & $\begin{array}{c}\text { Município } \\
\text { E }\end{array}$ \\
\hline $\begin{array}{l}\text { Fortalecimento da } \\
\text { atenção básica }\end{array}$ & $\mathrm{X}$ & & $\mathrm{X}$ & $\mathrm{X}$ & $\mathrm{X}$ \\
\hline $\begin{array}{l}\text { Fortalecimento das } \\
\text { ações da ESF }\end{array}$ & $\mathrm{X}$ & & & & \\
\hline $\begin{array}{l}\text { Aumento do número de } \\
\text { trabalhadores }\end{array}$ & & & $\mathrm{X}$ & & \\
\hline $\begin{array}{l}\text { Implantação de } \\
\text { prontuário eletrônico } \\
\text { unificado: hospital e } \\
\text { unidade sanitária }\end{array}$ & & & & $\mathrm{X}$ & \\
\hline
\end{tabular}


(Conclusão)

\begin{tabular}{|c|c|c|c|c|c|}
\hline Estratégias municipais & $\begin{array}{c}\text { Município } \\
\text { A }\end{array}$ & $\begin{array}{c}\text { Município } \\
\text { B }\end{array}$ & $\begin{array}{l}\text { Município } \\
\text { C }\end{array}$ & $\begin{array}{c}\text { Município } \\
\text { D }\end{array}$ & $\begin{array}{c}\text { Município } \\
\mathrm{E}\end{array}$ \\
\hline $\begin{array}{l}\text { Incentivo à educação } \\
\text { permanente }\end{array}$ & $x$ & $x$ & $\mathrm{x}$ & $x$ & $x$ \\
\hline $\begin{array}{l}\text { Aprimoramento da } \\
\text { vigilância e da } \\
\text { promoção da saúde }\end{array}$ & $\mathrm{x}$ & & $\mathrm{x}$ & $\mathrm{x}$ & $\mathrm{x}$ \\
\hline Adesão ao PQA-VS & & & & & $\mathrm{x}$ \\
\hline $\begin{array}{l}\text { Garantia do } \\
\text { atendimento integral }\end{array}$ & $x$ & & $x$ & $x$ & $x$ \\
\hline $\begin{array}{l}\text { Reorganização da } \\
\text { assistência } \\
\text { farmacêutica }\end{array}$ & $x$ & & $\mathrm{x}$ & $x$ & \\
\hline $\begin{array}{l}\text { Promoção da } \\
\text { alimentação saudável e } \\
\text { redução da obesidade }\end{array}$ & $x$ & & $x$ & $x$ & $\mathrm{x}$ \\
\hline $\begin{array}{l}\text { Qualificação do } \\
\text { acompanhamento de } \\
\text { gestantes e crianças e } \\
\text { incentivo ao } \\
\text { aleitamento materno } \\
\text { exclusivo }\end{array}$ & $\mathrm{x}$ & & $x$ & $x$ & $x$ \\
\hline $\begin{array}{l}\text { Implantação da Rede } \\
\text { Cegonha e PIM }\end{array}$ & & & $x$ & & \\
\hline $\begin{array}{l}\text { Incentivo à prática de } \\
\text { atividades físicas }\end{array}$ & & & & $x$ & \\
\hline $\begin{array}{l}\text { Projetos para redução } \\
\text { do consumo de álcool e } \\
\text { tabaco }\end{array}$ & $x$ & & $x$ & $x$ & $\mathrm{x}$ \\
\hline $\begin{array}{l}\text { Ampliação da cobertura } \\
\text { de exame preventivo de } \\
\text { câncer de colo uterino }\end{array}$ & $\mathrm{x}$ & & $\mathrm{x}$ & $\mathrm{x}$ & \\
\hline $\begin{array}{l}\text { Aumento da cobertura } \\
\text { de mamografia em } \\
\text { mulheres entre } 50 \text { e } 69 \\
\text { anos }\end{array}$ & & & $\mathrm{x}$ & $\mathrm{x}$ & \\
\hline
\end{tabular}

Fonte: Elaborado pelos autores - 2018

A definição de diretrizes, objetivos e metas está contemplada no PMS. Nessa seção do plano, devem estar estabelecidos os compromissos municipais e as estratégias de enfrentamento dos principais problemas de saúde no período de quatro anos. Entre as responsabilidades assumidas pelos gestores, prevaleceu o fortalecimento da atenção básica. Destacam-se a ampliação de recursos materiais e humanos e a qualificação do atendimento para aumentar sua resolutividade. O plano do município A ressaltou o 
fortalecimento das ações da ESF por meio de mapeamento, aumento de equipes e organização da atenção na área rural. Para o município C, a estratégia será aumentar o número de trabalhadores, e o município $\mathrm{D}$ pretende implantar prontuário eletrônico unificado entre hospital e unidade sanitária. O incentivo à educação permanente dos profissionais de saúde esteve entre as propostas unânimes.

Os PMS dos municípios A, C, D e E também sugeriram como meta, o aprimoramento da vigilância e da promoção da saúde por meio do controle, da notificação e da alimentação dos sistemas de informação. Já município E aderiu ao Programa de Qualidade e Avaliação da Vigilância em Saúde (PQA-VS).

Os municípios intencionaram o fortalecimento do vínculo dos profissionais com a comunidade, o desenvolvimento de ações educativas valorizando a promoção da saúde e o investimento na integralidade e na intersetorialidade por meio de parcerias. Os municípios A, C e D citaram como compromisso a reorganização da assistência farmacêutica, visando melhorar o acesso da população aos medicamentos básicos.

O Plano de Ações Estratégicas para o Enfrentamento das DCNT no Brasil (20112022) $)^{2}$ visa preparar o país para enfrentar as DCNT por meio de políticas públicas efetivas e integradas, diminuindo a morbimortalidade e a incapacidade direcionada ao grupo das quatro principais doenças e seus fatores de risco. ${ }^{2}$ Conforme demonstrado no Quadro 3, somente o município B não apresentou em seu PMS as metas deste plano. $O$ município D contemplou 75\% delas; A e C comprometeram-se com 50\%; e E propôs cumprir $41 \%$ das metas.

Quadro 3 - Propostas municipais relacionadas às metas de enfrentamento às DCNT no Brasil (2011-2022)

\begin{tabular}{|l|c|c|c|c|c|}
\hline $\begin{array}{c}\text { Metas nacionais do } \\
\text { Plano de Ações } \\
\begin{array}{c}\text { Estratégicas para o } \\
\text { Enfrentamento das }\end{array}\end{array}$ & \multicolumn{5}{|c|}{ Metas municipais } \\
\cline { 2 - 6 } $\begin{array}{c}\text { DCNT no Brasil (2011- } \\
\text { 2022) }\end{array}$ & $\begin{array}{c}\text { Município } \\
\text { A }\end{array}$ & $\begin{array}{c}\text { Município } \\
\text { B }\end{array}$ & $\begin{array}{c}\text { Município } \\
\text { C }\end{array}$ & $\begin{array}{c}\text { Município } \\
\text { D }\end{array}$ & $\begin{array}{c}\text { Município } \\
\text { E }\end{array}$ \\
\hline $\begin{array}{l}\text { Reduzir a taxa de } \\
\text { mortalidade prematura } \\
\text { (<70 anos) por DCNT } \\
\text { em 2\% ao ano }\end{array}$ & & $x$ & $X$ & $X$ & \\
\hline $\begin{array}{l}\text { Reduzir a prevalência } \\
\text { de obesidade em } \\
\text { crianças }\end{array}$ & $\mathrm{X}$ & & $\mathrm{X}$ & $\mathrm{X}$ & $\mathrm{X}$ \\
\hline
\end{tabular}


(Conclusão)

\begin{tabular}{|c|c|c|c|c|c|}
\hline \multirow{2}{*}{$\begin{array}{c}\text { Metas nacionais do } \\
\text { Plano de Ações } \\
\text { Estratégicas para o } \\
\text { Enfrentamento das } \\
\text { DCNT no Brasil (2011- } \\
\text { 2022) }\end{array}$} & \multicolumn{5}{|c|}{ Metas municipais } \\
\hline & $\begin{array}{c}\text { Município } \\
\text { A }\end{array}$ & $\begin{array}{c}\text { Município } \\
\text { B }\end{array}$ & $\begin{array}{l}\text { Município } \\
\text { C }\end{array}$ & $\begin{array}{c}\text { Município } \\
\text { D }\end{array}$ & $\begin{array}{c}\text { Município } \\
\text { E }\end{array}$ \\
\hline $\begin{array}{l}\text { Reduzir a prevalência } \\
\text { de obesidade em } \\
\text { adolescentes }\end{array}$ & $\mathrm{x}$ & & $x$ & $x$ & $x$ \\
\hline $\begin{array}{l}\text { Deter o crescimento da } \\
\text { obesidade em adultos }\end{array}$ & $x$ & & & $x$ & $x$ \\
\hline $\begin{array}{l}\text { Reduzir as prevalências } \\
\text { de consumo nocivo de } \\
\text { álcool }\end{array}$ & $x$ & & $x$ & $x$ & \\
\hline $\begin{array}{l}\text { Aumentar a prevalência } \\
\text { de atividade física no } \\
\text { lazer }\end{array}$ & & & & $x$ & \\
\hline \multicolumn{6}{|l|}{$\begin{array}{l}\text { Aumentar o consumo } \\
\text { de frutas e hortaliças }\end{array}$} \\
\hline $\begin{array}{l}\text { Reduzir o consumo } \\
\text { médio de sal }\end{array}$ & & & & & $x$ \\
\hline $\begin{array}{l}\text { Reduzir a prevalência } \\
\text { de tabagismo em } \\
\text { adultos }\end{array}$ & $\mathrm{x}$ & & & $x$ & $x$ \\
\hline $\begin{array}{l}\text { Aumentar a cobertura } \\
\text { de mamografia em } \\
\text { mulheres entre } 50 \text { e } 69 \\
\text { anos }\end{array}$ & & & $x$ & $x$ & \\
\hline $\begin{array}{l}\text { Ampliar a cobertura de } \\
\text { exame preventivo de } \\
\text { câncer de colo uterino } \\
\text { em mulheres de } 25 \text { a } 64 \\
\text { anos }\end{array}$ & $\mathrm{x}$ & & $x$ & $x$ & \\
\hline $\begin{array}{l}\text { Tratar } 100 \% \text { das } \\
\text { mulheres com } \\
\text { diagnóstico de lesões } \\
\text { precursoras de câncer }\end{array}$ & & & & & \\
\hline
\end{tabular}

Fonte: Elaborado pelos autores - 2018

Os municípios, A, C, D e E destacaram metas para a promoção da alimentação saudável e a redução da obesidade. Nas estratégias constam parceria com escolas e campanhas educativas à população. O município E propõe, em parceria com a Secretaria de Educação, a oferta de alimentação escolar com menor quantidade de sal. Nenhum município propôs metas para aumentar o consumo de frutas e hortaliças.

Os municípios propõem qualificar o acompanhamento de gestantes e crianças e 
incentivar o aleitamento materno exclusivo, o que em longo prazo favorece a redução da obesidade. O município C tem como meta a implantação da Rede Cegonha e PIM. Somente o município $D$ incluiu o incentivo à prática de atividades físicas. Os municípios incluíram projetos para a redução do consumo de álcool e tabaco, por meio da implementação de grupos e um dos PMS propõe a adesão ao programa Pulmão Ativo, de cessação e controle ao tabagismo.

Os PMS dos municípios A, C e D pretendem ampliar a cobertura de exame citopatológico de colo uterino em mulheres de 25 a 64 anos, e somente os municípios $C$ e D planejaram aumentar a cobertura de mamografia em mulheres entre 50 a 69 anos. Observou-se que nenhum dos PMS mencionaram o tratamento de $100 \%$ das mulheres com diagnóstico de lesões precursoras de câncer, proposto pelo plano nacional. ${ }^{2}$

\section{Debates na comissão intergestores regional relacionados às DCNT}

Analisaram-se 12 pautas e 14 atas das reuniões da CIR 29 de 2014, destacando os temas relacionados com o enfrentamento às DCNT, conforme dispostos no Quadro 4.

Quadro 4 - Pontos de pauta debatidos nas reuniões da CIR 29 de 2014

\begin{tabular}{|l|c|}
\hline \multicolumn{1}{|c|}{ Pontos de pauta } & Quantidade \\
\hline Construção das Academias de Saúde & 2 \\
\hline Disponibilidade de vacinas contra o Papilomavírus (HPV) & 1 \\
\hline Aquisição de ambulâncias e de veículos para transporte de pacientes & 6 \\
\hline Recursos materiais e equipamentos & 11 \\
\hline Reforma e ampliação de UBS & 11 \\
\hline Habilitação do Hospital Dia & 1 \\
\hline Participação em cursos e oficinas & 5 \\
\hline $\begin{array}{l}\text { Adesão e qualificação de projetos e de programas de enfrentamento às } \\
\text { DCNT }\end{array}$ & 17 \\
\hline Projetos de ação em saúde mental & 7 \\
\hline Educação em saúde coletiva & 2 \\
\hline Implantação da Política Estadual de Atenção Integral à Saúde do Homem & 2 \\
\hline Adesão ao PQA-VS & 4 \\
\hline Serviços de diagnóstico e de tratamento às pessoas com DCNT & 7 \\
\hline Articulação entre os componentes da rede & 1 \\
\hline
\end{tabular}

Fonte: Elaborado pelos autores - 2018

Como propostas para promoção da saúde, aparecem dois pedidos de aprovação 
de projetos para a construção de Academias da Saúde. Em uma das reuniões, servidores da 16a CRS anunciaram a disponibilidade de vacinas contra o Papilomavírus (HPV) para meninas de 11 a 13 anos.

A Portaria no $483 / 2014^{13}$ determina, como competência das três esferas de gestão, a garantia de infraestrutura, tecnologias adequadas e profissionais qualificados, a fim de prestar assistência às pessoas com DCNT - pontos predominantes nas pautas de reuniões da CIR 29. A aquisição de veículos para transportar pacientes esteve em seis atas e em 16 planos de trabalho apresentados por nove municípios. Os recursos materiais e equipamentos para UBS, hospitais e Unidade de Pronto Atendimento (UPA) e a reforma e ampliação de UBS, apareceram em 11 reuniões. Três municípios apresentaram projetos de instalação, reforma e contratação de profissionais para os CAPS. Destaca-se ainda a proposta de habilitação de um Hospital Dia e o projeto de construção do Ambulatório de Especialidades Médicas apresentado por uma Instituição de Ensino Superior (IES) da região.

Uma das diretrizes da PNPS é o apoio à formação e à educação permanente em saúde, buscando ampliar o compromisso e a capacidade crítica e reflexiva dos gestores e trabalhadores de saúde. ${ }^{12}$ Destaca-se a divulgação realizada pela 16a CRS sobre cursos de aperfeiçoamento do cuidado à saúde do adolescente e do idoso e sobre a Mostra Regional de Atenção Básica. Acrescenta-se um curso de especialização e a Conferência de Saúde do Trabalhador da Macrorregião dos Vales. O convite da 16a CRS para o encontro de construção dos PMS é outro exemplo de aperfeiçoamento das práticas de gestão em saúde.

Uma das competências municipais estabelecidas pela Portaria no 483/2014 ${ }^{13}$ é planejar ações e serviços para o cuidado das pessoas com DCNT. Os gestores debateram a adesão e a qualificação de programas associados ao enfrentamento das DCNT em nove reuniões e 17 pontos de pauta. Quatro municípios solicitaram adesão ao incentivo estadual para implantação de Oficinas Terapêuticas e, ainda, ao Encontro Regional em Saúde Mental, desenvolvido pela 16a CRS. Um município apresentou projeto para implantação do Núcleo de Apoio à Atenção Básica (NAAB) para qualificar as ações de Saúde Mental.

Consta nas atas a explanação sobre a Rede de Educação em Saúde Coletiva e o Projeto Caminhos do Cuidado por representante da 16a CRS. Houve também um pedido 
de adesão ao Núcleo Municipal de Educação em Saúde Coletiva e dois de implantação da Política Estadual de Atenção Integral à Saúde do Homem. A adesão ao PQA-VS foi pauta em quatro reuniões da CIR, solicitada por oito municípios.

Abordou-se assuntos referentes aos serviços de diagnóstico e de tratamento relacionados às DCNT. Entre eles, as cotas para cirurgia vascular, as transferências dos tetos financeiros de exames entre os municípios, os pedidos de habilitação aos serviços de urologia, a permanência da Rede de Atenção Oncológica e a solicitação de recursos pelo Serviço de Hemoterapia, totalizando sete pontos de pautas. Por fim, para aperfeiçoar a articulação entre os componentes da rede, a 16a CRS apresentou o modelo de documento de referência e contrarreferência para a Atenção de Média e Alta Complexidade.

\section{DISCUSSÃO}

O levantamento demográfico no PMS caracteriza a população, o número de habitantes, a distribuição em área urbana e rural, a raça e o sexo, o crescimento populacional, a pirâmide etária, as taxas de mortalidade e a esperança de vida ao nascer. ${ }^{14} \mathrm{~A}$ descrição sociodemográfica na análise situacional contribui para 0 reconhecimento de aspectos de vulnerabilidade. Dados referentes à razão de renda, Produto Interno Bruto (PIB), taxa de desemprego e escolaridade permitem caracterizar essa população e seus problemas, a fim de desenvolver ações de promoção da saúde. ${ }^{14}$ Verificou-se que o município E não contemplou essas informações na estrutura do PMS.

A análise epidemiológica no plano deve abranger dados de morbimortalidade, identificar o impacto dos problemas de saúde na população e desenvolver ações resolutivas a fim de reverter a situação. ${ }^{11} \mathrm{O}$ MS reforça que, para responder às mudanças nos padrões de adoecimento, o fortalecimento da vigilância da morbimortalidade e dos fatores de risco é prioridade nacional diante da epidemia das DCNT. ${ }^{2}$ Questionou-se a possibilidade de os municípios planejarem ações de promoção da saúde sem conhecer as causas do adoecimento e do óbito, pois dois municípios (B e E) não apresentaram dados epidemiológicos em seu PMS.

O potencial da promoção da saúde na prevenção das DCNT são fatores essenciais para as mudanças no sistema de saúde. Embora politicamente visível, a tradução do 
compromisso político em programas efetivos parece ser muito difícil. ${ }^{15}$

Expandir os serviços da Atenção Básica, estratégia proposta nos PMS, é fundamental para a melhoria do quadro epidemiológico, favorecendo a aproximação com a comunidade e o desenvolvimento de ações longitudinais de promoção da saúde. A PNPS12 reforça o planejamento de ações territorializadas e "reconhecendo os contextos locais e respeito às diversidades, favorecendo a construção de ambientes saudáveis e a busca da equidade e justiça social".

O fortalecimento da vigilância e o monitoramento às DCNT é uma das prioridades das três esferas de gestão, e deve-se considerar o esforço municipal em organizá-la para qualificar a assistência. ${ }^{2} \mathrm{O}$ avanço na ação intersetorial também se constitui como desafio, buscando articular ações de promoção da saúde na comunidade em projetos de melhoria na mobilidade urbana e na inclusão de idosos e pessoas com deficiência. ${ }^{16}$ Ambas as estratégias aparecem como propostas nos PMS. Também é possível observar a concordância dos municípios com as estratégias do MS quando propõem a ampliação do acesso gratuito aos insumos terapêuticos no cuidado às pessoas com DCNT. ${ }^{2}$

Observou-se que quatro municípios apresentaram no PMS metas relacionadas à alimentação saudável e à redução da obesidade. O risco de morbimortalidade aumenta conforme a elevação do Índice de Massa Corpórea (IMC), dobrando em pessoas com obesidade grave. ${ }^{17}$ São prioridades na PNPS ${ }^{12}$ ações relativas à alimentação saudável, visando à promoção da saúde e à segurança alimentar e nutricional, ao aconselhamento e à divulgação de práticas corporais e atividades físicas, incentivando a melhoria das condições dos espaços públicos e considerando a cultura local.

As atividades de promoção da saúde devem ser desenvolvidas da infância à fase adulta, com o objetivo de desenvolver hábitos saudáveis e prevenir doenças ocasionadas por má alimentação e sedentarismo. Ademais, as ações de promoção da saúde criadas na comunidade devem contar com a participação de diferentes áreas, como órgãos governamentais, escolas e serviços de saúde, fortalecendo o diálogo com a população e o comprometimento com os objetivos de cuidado propostos. ${ }^{2}$ Contudo, é preciso reconhecer que as práticas alimentares saudáveis são contra-hegemônicas em nossa sociedade e demandam estímulo e orientação continuados para que sejam adotadas no cotidiano das pessoas. ${ }^{18}$ 
Considerando a importante proporção da população rural nesses municípios, a ausência de metas quanto ao aumento do consumo de frutas e legumes causa estranheza, pois estes alimentos provavelmente são de fácil acesso à maioria da população. Isso pode estar relacionado à necessidade de mudança estrutural no sistema agroalimentar, que não será possível apenas por iniciativas individuais do setor produtivo, mas através de políticas públicas que possibilitem escolhas saudáveis. ${ }^{18}$

A proposta de implantação da Rede Cegonha e do PIM pode fortalecer as recomendações de hábitos de alimentação saudável na infância. A Rede Cegonha, conforme Portaria $\mathrm{n}$ o $1.459 / 2011,{ }^{19}$ quer garantir a atenção à mulher desde o planejamento reprodutivo, o pré-natal, o parto, o puerpério até o crescimento saudável da criança de 0 aos 24 meses. O PIM tem como objetivo a promoção do desenvolvimento integral da criança até os 5 anos de idade. ${ }^{20}$

Estudo apontou que as metas dos Objetivos de Desenvolvimento do Milênio para a saúde materno-infantil estão relacionadas às DCNT: o diabetes e a hipertensão na gestação incidem sobre o risco de morte materna e a falta do pré-natal bem conduzido poderá contribuir com a desnutrição intraútero, favorecendo o desenvolvimento de DCNT na fase adulta, e a ausência do aleitamento na infância pode propiciar a obesidade e a DCNT. ${ }^{21}$

Destaca-se que somente um PMS apresentou proposta de incentivar atividade física. O estímulo a essa prática está vinculado a ações intersetoriais e ao planejamento urbano, com remodelação do espaço físico visando à construção de ambientes saudáveis e seguros, reduzindo as iniquidades sociais. ${ }^{16}$ Nesse sentido, a promoção da saúde na perspectiva intersetorial envolve desafios, como a transposição de questões políticas e interesses pessoais, articulando a administração pública. A mobilização de outros campos de saber e atividade profissional amplia as possibilidades de enfrentamento dos determinantes socioambientais e favorece a promoção da saúde. ${ }^{22}$

Os municípios contemplaram em seu PMS a redução do consumo de álcool e tabaco. Este é o responsável por um grande número de óbitos e internações por ano relacionado ao câncer e a doenças do aparelho circulatório e respiratório. ${ }^{2}$ A PNPS inclui ações educativas, legislativas, econômicas, ambientais e sociais para o enfrentamento do uso do tabaco e seus derivados, assim como o uso abusivo de álcool e outras drogas. ${ }^{12}$ 
O diagnóstico precoce, em relação ao câncer de colo de útero e de mama, pode resultar em melhora da sobrevida da paciente. Portanto, a melhora do investimento no rastreamento dessas patologias é uma estratégia para a diminuição das DCNT. ${ }^{2}$ Três municípios apresentaram proposta de aumento na cobertura de citopatológico de colo uterino no PMS. Preocupante o fato de que somente dois municípios propuseram metas relacionadas ao diagnóstico precoce do câncer de mama e nenhum em relação ao seu tratamento. Considerando que o câncer de mama é a principal causa de morte por câncer em mulheres, o diagnóstico precoce e a garantia da referência para tratamento em tempo oportuno são fundamentais na diminuição da mortalidade por esse tipo de câncer. As estratégias para a atenção às mulheres com câncer na rede de saúde precisam estar contempladas nos PMS.

Observou-se uma grande diversidade de temas relacionados às DCNT debatidos na CIR, o que se justifica pela complexidade das ações necessárias para seu controle. A Portaria no 483/2014, ${ }^{13}$ que redefine a Rede de Atenção à Saúde das Pessoas com Doenças Crônicas e a organização das linhas de cuidado, visa à atenção integral à saúde. Nesse sentido, uma das propostas são as Academias da Saúde, iniciativa do MS estabelecida pela Portaria no 2.681/2013 e também no Plano de Ações Estratégicas para o Enfrentamento das DCNT no Brasil, considerando que a atividade física regular reduz o risco de doença circulatória, inclusive de hipertensão, diabetes mellitus, câncer de mama e cólon, bem como da depressão. ${ }^{2,23}$

O câncer de colo uterino é o quarto tipo de câncer mais comum entre as mulheres. O principal fator de risco para seu desenvolvimento é a infecção pelo HPV. Assim, o MS incluiu no seu calendário a vacinação contra esse vírus, ${ }^{24}$ um dos temas discutidos na CIR.

Os modelos inovadores de prevenção e controle das DCNT devem levar em consideração os diversos ciclos de vida. ${ }^{25} \mathrm{O}$ MS sugere a implementação de programas de promoção da saúde que proporcionem cuidados no pré-natal, acompanhamento da nutrição da gestante, controle de hipertensão e dos níveis de açúcar no sangue, bem como o incentivo ao aleitamento materno. ${ }^{2}$ No entanto, estudo recente aponta que as ações de promoção da saúde e prevenção de DCNT pelas equipes de atenção primária no país são insuficientes. Isso já denota a urgência na investigação das razões para essa situação e a ampliação das ações preconizadas pela PNPS. ${ }^{26}$ 
O aleitamento exclusivo por 6 meses e complementado até os 2 anos ou mais proporciona benefícios em longo prazo, como a proteção contra o diabetes mellitus tipo II, além de reduzir as chances de a criança apresentar sobrepeso quando adulta. ${ }^{27}$ Essa proteção estende-se ao longo da infância e da adolescência quanto à exposição aos fatores de risco (álcool e tabaco) e ao estímulo dos fatores protetores, alimentação saudável e atividade física, persistindo até a vida adulta. ${ }^{2}$

As doenças mentais estão entre as principais causas de incapacidade no mundo, e conforme estimativas, até 2020 só serão superadas pelas doenças cardíacas. Assim, a atenção à saúde mental deve estar entre os planos de enfrentamento às DCNT com a construção de estratégias de prevenção e monitoramento dessas doenças. ${ }^{28}$

O plano de enfrentamento das DCNT parte do princípio de que ações de prevenção e controle exigem articulação e apoio de setores do governo, da sociedade civil e do setor privado. ${ }^{29}$ A PNPS $^{12}$ traz a intersetorialidade como princípio, referindo-se ao "processo de articulação de saberes, potencialidades e experiências de sujeitos, grupos e setores na construção de intervenções compartilhadas, estabelecendo vínculos, corresponsabilidade e cogestão para objetivos comuns".

Nesse sentido, espera-se avançar nas ações de articulação intersetorial para o enfrentamento das DCNT, transversal aos processos de organização dos serviços de saúde. Os desafios em relação à essa articulação serão minimizados com a participação da sociedade civil, de entidades da saúde, de universidades, dos Conselhos de Saúde, das ONG e dos usuários do SUS. ${ }^{30}$

\section{CONSIDERAÇÕES FINAIS}

O PMS é um dos instrumentos centrais no processo de planejamento devido à sua relevância no levantamento de problemas na saúde da população e na elaboração de estratégias para solucioná-los. Verificou-se que os municípios reconhecem sua importância, assim como a necessidade de ações intersetoriais.

A análise situacional de saúde apareceu em todos os planos, evidenciando as características populacionais e a identificação de fatores condicionantes para o aumento das DCNT, como a mudança do perfil demográfico. Verificou-se que não há um padrão entre os municípios, contemplando alguns dos aspectos relevantes e 
negligenciando outros, como a promoção da saúde.

A vigilância e a promoção da saúde são estratégias para o controle das DCNT. Observou-se a falta de dados importantes em vários municípios, como o perfil de morbimortalidade, fato não justificável, pois há fácil acesso às fontes de informações em saúde. Constatou-se a ausência da descrição da gestão em saúde, de monitoramento e de avaliação do sistema, comprometendo o planejamento municipal em saúde. Esse cenário pode ser um indicativo das dificuldades dos gestores e de suas equipes para o uso adequado dos sistemas de informação disponíveis como recursos importantes no planejamento e na gestão da saúde.

Em relação às metas nacionais, a maioria consta nos planos, destacando-se atividades de promoção da saúde que reduzam o índice de obesidade, bem como propostas para a diminuição do consumo de tabaco e álcool, e de ampliação do exame preventivo de câncer de colo de útero. Questiona-se a qualidade da promoção da saúde do município em que não houve propostas de melhoria nesse âmbito em seu PMS.

Identificou-se debates relacionados ao enfrentamento das DCNT no âmbito do SUS na maioria dos pontos de pauta e das atas das reuniões da CIR 29, embora pouco relacionados à promoção da saúde. Considera-se relevante o desenvolvimento da rede articulada e de linhas de cuidado às DCNT, além de manter a atenção sobre as metas encaminhadas, ampliar as ações implementadas e rever estratégias para os pontos que não mostraram avanços. Ações e programas previstos no planejamento devem contemplar cuidados que permitam a promoção da saúde e a prevenção de fatores de risco, dispondo da atenção básica como principal porta de entrada do SUS e coordenadora do processo de cuidado.

\section{REFERÊNCIAS}

1. Ministério da Saúde (MS). Saúde Brasil 2011: uma análise da situação de saúde e a vigilância da saúde da mulher. Brasília: MS; 2012.

2. Ministério da Saúde (MS). Plano de ações estratégicas para o enfrentamento das doenças crônicas não transmissíveis (DCNT) no Brasil 2011-2022. Brasília: MS; 2011.

3. Ministério da Saúde (MS). Diretrizes e recomendações para o cuidado integral de doenças crônicas não-transmissíveis: promoção da saúde, vigilância, prevenção e assistência. Brasília: MS; 2008. 
4. Moysés ST, Sá RF. Planos locais de promoção da saúde: intersetorialidade(s) construída(s) no território. Ciênc. Saúde Coletiva. 2014; 19(11):4323-30.

5. Paim JS. Planejamento em saúde para não especialistas. In: Campos GWS, organizador. Tratado de Saúde Coletiva. São Paulo: Hucitec; 2013. p. 827-843.

6. Brasil. Decreto no 7.508, de 28 de junho de 2011. Regulamenta a Lei no 8.080, de 19 de setembro de 1990, para dispor sobre a organização do Sistema Único de Saúde - SUS, o planejamento da saúde, a assistência à saúde e a articulação interfederativa, e dá outras providências. Diário Oficial da União 2011; 29 jun.

7. Brasil. Portaria $\mathrm{n} 02.135$, de 25 de setembro de 2013. Estabelece diretrizes para o processo de planejamento no âmbito do Sistema Único de Saúde (SUS). Diário Oficial da União 2013; 25 set.

8. Oliveira-Campos M, Rodrigues-Neto JF, Silveira MF, Neves DMR, Vilhena JM, Oliveira JF, Magalhães JC, Drumond D. Impacto dos fatores de risco para doenças crônicas não transmissíveis na qualidade de vida. Ciênc. Saúde Coletiva 2013;18(3):873-82.

9. Instituto Brasileiro de Geografia e Estatística [Homepage na Internet]. Censo Demográfico de 2010 [acessado em 2014 Out 04]. Disponível em: http://www.ibge.gov.br/home/estatistica/populacao/censo2010/.

10. Brasil. Departamento de Informática do SUS. Informações em Saúde. 2015. [acessado em 2015 Out 15]. Disponível em: http://tabnet.datasus.gov.br/cgi/dh.exe?pacto/2010/cnv/pactrs.def.

11. Teixeira CF, Vilasbôas ALQ, Jesus WLA. Proposta metodológica para o planejamento no Sistema Único de Saúde. In: Teixeira CF, organizador. Planejamento em saúde: conceitos, métodos e experiências. Salvador: EDUFBA; 2010. p. 51-75.

12. Brasil. Portaria no 2.446, de 11 de novembro de 2014. Redefine a Política Nacional de Promoção da Saúde (PNPS). Diário Oficial da União 2014; 11 nov.

13. Brasil. Portaria 483, de 10 de abril de 2014. Redefine a rede de atenção à saúde das pessoas com doenças crônicas no âmbito do Sistema Único de Saúde (SUS) e estabelece diretrizes para a organização das suas linhas de cuidado. Diário Oficial da União 2014; 10 abr.

14. Ministério da Saúde (MS). Sistema de planejamento do SUS: uma construção coletiva: instrumentos básicos. 2. ed. Brasília: MS; 2009.

15. Zaletel J, Piletic M, Lindström J, Icks A, Rothe U, Sørensen M, Maggini M. National Diabetes Plans: can they support changes in health care systems to strengthen diabetes prevention and care? Ann Ist Super Sanità 2015; 51(3):206-8.

16. Malta DC, Silva MMA, Albuquerque GM, Lima CM, Cavalcante $T$, Jaime PC, Júnior JBS. A implementação das prioridades da Política Nacional de Promoção da Saúde, um balanço, 2006 a 2014. Ciênc. Saúde Coletiva 2014; 19(11):4301-11.

17. Kivimäki M, Kuosma E, Ferrie JE, Luukkonen R, Nyberg ST, Alfredsson L, Batty GD, Brunner EJ, Fransson E, Goldberg M, Knutsson A, Koskenvuo M, Nordin M, Oksanen T, Pentti J, Rugulies R, Shipley MJ, Singh-Manoux A, Steptoe A, Suominen 
SB, Theorell T, Vahtera J, Virtanen M, Westerholm P, Westerlund H, Zins M, Hamer $\mathrm{M}$, Bell JA, Tabak AG, Jokela M. Overweight, obesity, and risk of cardiometabolic multimorbidity: pooled analysis of individual-level data for 120813 adults from 16 cohort studies from the USA and Europe. Lancet Public Health 2017; 2:277-85.

18. Castro IRR. Challenges and perspectives for the promotion of adequate and healthy food in Brazil. Cad. Saúde Pública 2015; 31(1):7-9.

19. Brasil. Portaria no 1.459, de 24 de junho de 2011. Institui no âmbito do Sistema Único de Saúde - SUS - a Rede Cegonha. Diário Oficial da União 2011; 24 jun.

20. Brasil. Lei no 12.544, de 03 de julho de 2006. Institui o Programa Primeira Infância Melhor - PIM - e dá outras providências. Diário Oficial da União 2006; 03 jul.

21. Beaglehole R, Bonita R, Horton R, Ezzati M, Bhala N, Amuyunzu-Nyamongo M, Modi M, Reddy KS. Measuring progress on NCDs: one goal and five targets. Lancet Public Health 2012; 380 (9850):1283-5.

22. Silva KL, Sena RR, Akerman M, Belga SMM, Rodrigues AT. Intersetorialidade, determinantes socioambientais e promoção da saúde. Ciênc. Saúde Coletiva 2014; 19(11):4361-70.

23. Brasil. Portaria $\mathrm{n}$ - 2.681, de 07 de novembro de 2013. Redefine o Programa Academia da Saúde no âmbito do Sistema Único de Saúde (SUS). Diário Oficial da União 2013; 07 nov.

24. Instituto Nacional de Câncer José Alencar Gomes da Silva [Homepage na Internet]. [Acessado em 2015 Abr 09]. Disponível em: http://www.inca.gov.br/estimativa/2014/sintese-de-resultados-comentarios.asp.

25. Goulart FAA. Doenças crônicas não transmissíveis: estratégias de controle e desafios para os sistemas de saúde. Brasília: Ministério da Saúde; 2011.

26. Medina MG, Aquino R, Vilasbôas ALQ, Mota E, Júnior EPP, Luz LA, Anjos DSO, Pinto ICM. Promoção da saúde e prevenção de doenças crônicas: o que fazem as equipes de Saúde da Família? Saúde Debate 2014; 38(no especial):69-82.

27. Ministério da Saúde (MS). Saúde da criança: nutrição infantil: aleitamento materno e alimentação complementar. Brasília: MS; 2009.

28. Organização Mundial da Saúde (OMS). Cuidados inovadores para condições crônicas: componentes estruturais de ação: relatório mundial. Brasília: OMS; 2003. [Acessado 2015 mar 14]. Disponível em: https://www.opas.org.br/wpcontent/uploads/2015/09/Manual_final.pdf.

29. Malta DC, Merhy EE. O percurso da linha do cuidado sob a perspectiva das doenças crônicas não transmissíveis. Interface - Comunic, Saúde, Educ. 2010; 14(34):593-603.

30. Malta DC, Oliveira TP, Santos MAS, Andrade SSCA, Silva MMA. Avanços do Plano de Ações Estratégicas para o Enfrentamento das Doenças Crônicas não Transmissíveis no Brasil, 2011-2015. Epidemiol. Serv. Saúde. 2016; 25(2):373-90. 
Submissão: maio de 2018.

Aprovação: novembro de 2018. 